Vietnam Journal of Mechanics, VAST, Vol.34, No. 2 (2012), pp. $91-99$

\title{
NUMERICAL CALCULATING LINEAR VIBRATIONS OF THIRD ORDER SYSTEMS INVOLVING FRACTIONAL OPERATORS
}

\author{
Nguyen Van Khang ${ }^{1}$, Tran Dinh Son ${ }^{2}$, Bui Thi Thuy ${ }^{2}$ \\ ${ }^{1}$ Hanoi University of Technology, Vietnam \\ ${ }^{2}$ Hanoi University of Mining and Geology, Vietnam
}

\begin{abstract}
This paper presents a numerical method for dynamic calculation of third order systems involving fractional operators. Using the Liouville-Rieman's definition of fractional derivatives, a numerical algorithm is developed on base of the well-known Newmark integration method to calculate dynamic response of third order systems. Then, we apply this method to calculate linear vibrations of viscoelastic systems containing fractional derivatives.
\end{abstract}

Key words: Fractional order derivative, numerical method, vibration, third order system.

\section{INTRODUCTION}

In 1959 Newmark presented a family of single-step integration methods for the solution of structural dynamic problems [1,2]. During the past time Newmark's method has been applied to the dynamic analysis of many practical engineering structures. It has been modified and improved by many other researchers such as Wilson, Hilber, Hughes and Taylor... However, these methods are only used for the system of second order equations.

The concepts of fractional derivatives $[3,4,5]$ appeared many years ago and are introduced by famous mathematicians like Riemann, Liouville, Grünwald, Letnikov, Caputo... The concept of fractional operators in engineering applications is now increasingly attractive in the formulations of the constitutive law for some viscoelastic materials.

In $[6,7,8]$ Shimizu and Zhang have used the Newmark integration method for calculating the vibrations of second order systems involving fractional derivatives. Many vibration problems in engineering lead the system of differential equations of third order. In this paper we present the using Newmark integration method for calculating vibrations of third order systems involving fractional derivatives.

\section{THE NEWMARK METHOD FOR THE THIRD ORDER SYSTEMS}

The Newmark method is a single-step integration formula. The state vector of the system at a time $t_{n+1}=t_{n}+h$ is deduced from the already-known state vector at time $t_{n}$ 
through a Taylor expansion of the displacements, velocities and accelerations

$$
\boldsymbol{f}\left(t_{n}+h\right)=\boldsymbol{f}\left(t_{n}\right)+h \dot{\boldsymbol{f}}\left(t_{n}\right)+\frac{h^{2}}{2 !} \ddot{\boldsymbol{f}}\left(t_{n}\right)+\ldots+\frac{h^{s}}{s !} \boldsymbol{f}^{(s)}\left(t_{n}\right)+\boldsymbol{R}_{s},
$$

where $\boldsymbol{R}_{s}$ is the remainder of the development to the order $s$

$$
\boldsymbol{R}_{s}=\frac{1}{s !} \int_{t_{n}}^{t_{n}+h} \boldsymbol{f}^{(s+1)}(\tau)\left[t_{n}+h-\tau\right]^{s} d \tau .
$$

Relation (1) allows us to compute the accelerations, velocities and displacements of a system at time $t_{n+1}$

$$
\begin{gathered}
\ddot{\boldsymbol{q}}_{n+1}=\ddot{\boldsymbol{q}}_{n}+\int_{t_{n}}^{t_{n+1}} \dddot{\boldsymbol{q}}(\tau) d \tau \\
\dot{\boldsymbol{q}}_{n+1}=\dot{\boldsymbol{q}}_{n}+h \ddot{\boldsymbol{q}}_{n}+\int_{t_{n}}^{t_{n+1}}\left(t_{n+1}-\tau\right) \dddot{\boldsymbol{q}}(\tau) d \tau, \\
\boldsymbol{q}_{n+1}=\boldsymbol{q}_{n}+h \dot{\boldsymbol{q}}_{n}+\frac{h^{2}}{2} \ddot{\boldsymbol{q}}_{n}+\frac{1}{2} \int_{t_{n}}^{t_{n+1}}\left(t_{n+1}-\tau\right)^{2} \dddot{\boldsymbol{q}}(\tau) d \tau .
\end{gathered}
$$

Let us express $\dddot{\boldsymbol{q}}(\tau)$ in the time interval $\left[t_{n}, t_{n+1}\right]$ as a function of $\dddot{\boldsymbol{q}}_{n}, \dddot{\boldsymbol{q}}_{n+1}$ at the interval limits

$$
\begin{aligned}
\dddot{\boldsymbol{q}}_{n} & =\dddot{\boldsymbol{q}}(\tau)+\boldsymbol{q}^{(4)}(\tau)\left(t_{n}-\tau\right)+\boldsymbol{q}^{(5)}(\tau) \frac{\left(t_{n}-\tau\right)^{2}}{2}+\ldots \\
\dddot{\boldsymbol{q}}_{n+1} & =\dddot{\boldsymbol{q}}(\tau)+\boldsymbol{q}^{(4)}(\tau)\left(t_{n+1}-\tau\right)+\boldsymbol{q}^{(5)}(\tau) \frac{\left(t_{n+1}-\tau\right)^{2}}{2}+\ldots
\end{aligned}
$$

By multiplying the first equation of $(6)$ by $(1-\alpha)$, the second equation by $\alpha$ and adding two equations then, we obtain

$$
\dddot{\boldsymbol{q}}(\tau)=(1-\alpha) \dddot{\boldsymbol{q}}_{n}+\alpha \dddot{\boldsymbol{q}}_{n+1}+\boldsymbol{q}^{(4)}(\tau)\left[\tau-\alpha h-t_{n}\right]+\mathrm{O}\left(h^{2} \boldsymbol{q}^{(5)}\right) .
$$

Likewise, multiplying equations (6) by $(1-2 \gamma), 2 \gamma$ and by $(1-6 \beta), 6 \beta$ yields

$$
\begin{aligned}
& \dddot{\boldsymbol{q}}(\tau)=(1-2 \gamma) \dddot{\boldsymbol{q}}_{n}+2 \gamma \dddot{\boldsymbol{q}}_{n+1}+\boldsymbol{q}^{(4)}(\tau)\left[\tau-2 \gamma h-t_{n}\right]+\mathrm{O}\left(h^{2} \boldsymbol{q}^{(5)}\right) . \\
& \dddot{\boldsymbol{q}}(\tau)=(1-6 \beta) \dddot{\boldsymbol{q}}_{n}+6 \beta \dddot{\boldsymbol{q}}_{n+1}+\boldsymbol{q}^{(4)}(\tau)\left[\tau-6 \beta h-t_{n}\right]+\mathrm{O}\left(h^{2} \boldsymbol{q}^{(5)}\right) .
\end{aligned}
$$

Hence, by substituting (7), (8) and (9) in the integral terms of (3), (4) and (5), we obtain the quadrature formulas

$$
\int_{t_{n}}^{t_{n+1}} \dddot{\boldsymbol{q}}(\tau) d \tau=(1-\alpha) h \dddot{\boldsymbol{q}}_{n}+\alpha h \dddot{\boldsymbol{q}}_{n+1}+\boldsymbol{r}_{n},
$$




$$
\begin{gathered}
\int_{t_{n}}^{t_{n+1}}\left(t_{n+1}-\tau\right) \dddot{\boldsymbol{q}}(\tau) d \tau=\left(\frac{1}{2}-\gamma\right) h^{2} \dddot{\boldsymbol{q}}_{n}+\gamma h^{2} \dddot{\boldsymbol{q}}_{n+1}+\boldsymbol{r}_{n}^{\prime}, \\
\frac{1}{2} \int_{t_{n}}^{t_{n+1}}\left(t_{n+1}-\tau\right)^{2} \dddot{\boldsymbol{q}}(\tau) d \tau=\left(\frac{1}{6}-\beta\right) h^{3} \dddot{\boldsymbol{q}}_{n}+\beta h^{3} \dddot{\boldsymbol{q}}_{n+1}+\boldsymbol{r}_{n}^{\prime \prime},
\end{gathered}
$$

The corresponding error measure

$$
\begin{aligned}
& \boldsymbol{r}_{n}=\left(\alpha-\frac{1}{2}\right) h^{2} \boldsymbol{q}^{(4)}(\tilde{\tau})+\mathrm{O}\left(h^{3} \boldsymbol{q}^{(5)}\right), \\
& \boldsymbol{r}_{n}^{\prime}=\left(\gamma-\frac{1}{6}\right) h^{3} \boldsymbol{q}^{(4)}(\tilde{\tau})+\mathrm{O}\left(h^{4} \boldsymbol{q}^{(5)}\right), \quad t_{n}<\tilde{\tau}<t_{n+1} \\
& \boldsymbol{r}_{n}^{\prime \prime}=\left(\beta-\frac{1}{24}\right) h^{4} \boldsymbol{q}^{(4)}(\tilde{\tau})+\mathrm{O}\left(h^{5} \boldsymbol{q}^{(5)}\right) .
\end{aligned}
$$

The constants $\alpha, \gamma$ and $\beta$ are parameters associated with the quadrate scheme. Choosing values $\alpha=\frac{1}{2}, \gamma=\frac{1}{6}, \beta=\frac{1}{24}$ leads to linear interpolation of $\dddot{\boldsymbol{q}}(\tau)$

$$
\dddot{\boldsymbol{q}}(\tau)=\dddot{\boldsymbol{q}}_{n}+\left(\tau-t_{n}\right) \frac{\dddot{\boldsymbol{q}}_{n+1}-\dddot{\boldsymbol{q}}_{n}}{h},
$$

If we choose $\alpha=\frac{1}{2}, \gamma=\frac{1}{4}, \beta=\frac{1}{12}$, we obtain the average value of $\dddot{\boldsymbol{q}}(\tau)$ over the time interval $\left[t_{n}, t_{n+1}\right]$

$$
\dddot{\boldsymbol{q}}(\tau)=\frac{\dddot{\boldsymbol{q}}_{n}+\dddot{\boldsymbol{q}}_{n+1}}{2} .
$$

By substituting integrals (10), (11) and (12) into equations (3), (4) and (5), we get the approximation formulas of displacements, velocities and accelerations of system at time $t_{n+1}$

$$
\begin{gathered}
\ddot{\boldsymbol{q}}_{n+1}=\ddot{\boldsymbol{q}}_{n}+(1-\alpha) h \dddot{\boldsymbol{q}}_{n}+\alpha h \dddot{\boldsymbol{q}}_{n+1}, \\
\dot{\boldsymbol{q}}_{n+1}=\dot{\boldsymbol{q}}_{n}+h \ddot{\boldsymbol{q}}_{n}+\left(\frac{1}{2}-\gamma\right) h^{2} \dddot{\boldsymbol{q}}_{n}+\gamma h^{2} \dddot{\boldsymbol{q}}_{n+1}, \\
\boldsymbol{q}_{n+1}=\boldsymbol{q}_{n}+h \dot{\boldsymbol{q}}_{n}+\frac{h^{2}}{2} \ddot{\boldsymbol{q}}_{n}+\left(\frac{1}{6}-\beta\right) h^{3} \dddot{\boldsymbol{q}}_{n}+\beta h^{3} \dddot{\boldsymbol{q}}_{n+1} .
\end{gathered}
$$

Thus, we have established the approximation formulas (14), (15), (16) to approach solving the system of third order differential equations.

Let us then assume that the equations of dynamics

$$
\boldsymbol{M} \dddot{\boldsymbol{q}}+\boldsymbol{B} \ddot{\boldsymbol{q}}+\boldsymbol{C} \dot{\boldsymbol{q}}+\boldsymbol{K} \boldsymbol{q}=\boldsymbol{f}(t),
$$

are linear, i.e., that matrices $\boldsymbol{M}, \boldsymbol{B}, \boldsymbol{C}$ and $\boldsymbol{K}$ are independent of $\boldsymbol{q}$, and let us introduce the numerical scheme (14), (15) and (16) in the equations of motion at time $t_{n+1}$ so as to 
compute $\dddot{\boldsymbol{q}}_{n+1}$

$$
\begin{aligned}
& {\left[\boldsymbol{M}+\alpha h \boldsymbol{B}+\gamma h^{2} \boldsymbol{C}+\beta h^{3} \boldsymbol{K}\right] \dddot{\boldsymbol{q}}_{n+1}=\boldsymbol{f}_{n+1}-\boldsymbol{B}\left[\ddot{\boldsymbol{q}}_{n}+(1-\alpha) h \dddot{\boldsymbol{q}}_{n}\right]} \\
& -\boldsymbol{C}\left[\dot{\boldsymbol{q}}_{n}+h \ddot{\boldsymbol{q}}_{n}+\left(\frac{1}{2}-\gamma\right) h^{2} \dddot{\boldsymbol{q}}_{n}\right]-\boldsymbol{K}\left[\boldsymbol{q}_{n}+h \dot{\boldsymbol{q}}_{n}+\frac{h^{2}}{2} \ddot{\boldsymbol{q}}_{n}+\left(\frac{1}{6}-\beta\right) h^{3} \dddot{\boldsymbol{q}}_{n}\right] .
\end{aligned}
$$

By solving the system of linear equations (18) we obtain $\dddot{\boldsymbol{q}}_{n+1}$. Then, by using Newmark formulas (14), (15) and (16) we get accelerations, velocities and displacements $\ddot{\boldsymbol{q}}_{n+1}, \dot{\boldsymbol{q}}_{n+1}$ and $\boldsymbol{q}_{n+1}$. We determine the initial conditions of $\dddot{\boldsymbol{q}}\left(t_{0}\right)$ from the given values of $\boldsymbol{q}\left(t_{0}\right), \dot{\boldsymbol{q}}\left(t_{0}\right)$ and $\ddot{\boldsymbol{q}}\left(t_{0}\right)$

$$
\dddot{\boldsymbol{q}}\left(t_{0}\right)=\boldsymbol{M}^{-1}\left[\boldsymbol{f}\left(t_{0}\right)-\boldsymbol{B} \ddot{\boldsymbol{q}}\left(t_{0}\right)-\boldsymbol{C} \dot{\boldsymbol{q}}\left(t_{0}\right)-\boldsymbol{K} \boldsymbol{q}\left(t_{0}\right)\right] .
$$

Let us assume that the non-linear dynamic equations of third order systems have the following form

$$
\boldsymbol{M}(\boldsymbol{q}) \dddot{\boldsymbol{q}}+\boldsymbol{k}(t, \boldsymbol{q}, \dot{\boldsymbol{q}}, \ddot{\boldsymbol{q}})=\boldsymbol{f}(t, \boldsymbol{q}, \dot{\boldsymbol{q}}, \ddot{\boldsymbol{q}}),
$$

We have $\dddot{\boldsymbol{q}}_{n+1}$ from equation (16)

$$
\dddot{\boldsymbol{q}}_{n+1}=\frac{1}{\beta h^{3}}\left(\boldsymbol{q}_{n+1}-\boldsymbol{q}_{n}\right)-\frac{1}{\beta h^{2}} \dot{\boldsymbol{q}}_{n}-\frac{1}{2 \beta h} \ddot{\boldsymbol{q}}_{n}-\left(\frac{1}{6 \beta}-1\right) \dddot{\boldsymbol{q}}_{n}
$$

By substituting (21) into equations (14) and (15), we obtain

$$
\begin{gathered}
\dot{\boldsymbol{q}}_{n+1}=\frac{\gamma}{\beta h}\left(\boldsymbol{q}_{n+1}-\boldsymbol{q}_{n}\right)+\left(1-\frac{\gamma}{\beta}\right) \dot{\boldsymbol{q}}_{n}+\left(1-\frac{\gamma}{2 \beta}\right) h \ddot{\boldsymbol{q}}_{n}+\left(\frac{1}{2}-\frac{\gamma}{6 \beta}\right) h^{2} \dddot{\boldsymbol{q}}_{n} \\
\ddot{\boldsymbol{q}}_{n+1}=\frac{\alpha}{\beta h^{2}}\left(\boldsymbol{q}_{n+1}-\boldsymbol{q}_{n}\right)-\frac{\alpha}{\beta h} \dot{\boldsymbol{q}}_{n}+\left(1-\frac{\alpha}{2 \beta}\right) \ddot{\boldsymbol{q}}_{n}+\left(1-\frac{\alpha}{6 \beta}\right) h \dddot{\boldsymbol{q}}_{n} .
\end{gathered}
$$

We realize that $\dddot{\boldsymbol{q}}_{n+1}, \ddot{\boldsymbol{q}}_{n+1}, \dot{\boldsymbol{q}}_{n+1}$ are represented by $\boldsymbol{q}_{n+1}$ and the known values of $\boldsymbol{q}_{n}, \dot{\boldsymbol{q}}_{n}, \ddot{\boldsymbol{q}}_{n}, \dddot{\boldsymbol{q}}_{n}$. By substituting $\dddot{\boldsymbol{q}}_{n+1}, \ddot{\boldsymbol{q}}_{n+1}, \dot{\boldsymbol{q}}_{n+1}$ into (20), we obtain the system of non-linear algebraic equations with unknown $\boldsymbol{q}_{n+1}$. We have values of $\boldsymbol{q}_{n+1}$ through the Newton iteration method. Then, from equations (21), (22) and (23) we determine values of $\dot{\boldsymbol{q}}_{n+1}, \ddot{\boldsymbol{q}}_{n+1}$ and $\dddot{\boldsymbol{q}}_{n+1}$ with the initial conditions of $\dddot{\boldsymbol{q}}\left(t_{0}\right)$ derived from the equations of dynamics (20)

$$
\dddot{\boldsymbol{q}}_{0}=\boldsymbol{M}^{-1}\left(\boldsymbol{q}_{0}\right)\left[f\left(t_{0}, \boldsymbol{q}_{0}, \dot{\boldsymbol{q}}_{0}, \ddot{\boldsymbol{q}}_{0}\right)-\boldsymbol{k}\left(t_{0}, \boldsymbol{q}_{0}, \dot{\boldsymbol{q}}_{0}, \ddot{\boldsymbol{q}}_{0}\right)\right]
$$

\section{CALCULATING LINEAR VIBRATIONS OF THIRD ORDER SYSTEMS INVOLVING FRACTIONAL OPERATORS}

Consider now the motion differential equation of third order systems involving fractional derivative of order $q$

$$
\dddot{x}(t)+a \ddot{x}(t)+b D^{q} x(t)+c x(t)=f(t), \quad(0<q<1)
$$

where $a, b$, and $c$ are coefficients; $x(t)$ is the displacement of oscillator and $D^{q} x(t)$ represents the fractional derivative of order $q$. 
The Liouville - Riemann's fractional derivative is defined as $[3,4,5]$

$$
D^{q} x(t)=D\left[D^{-u} x(t)\right]=\frac{1}{\Gamma(u)} \frac{d}{d t} \int_{0}^{t} \frac{x(\tau)}{(t-\tau)^{1-u}} d \tau
$$

where $u=1-q, 0<u<1$.

In order to make use of Liouville - Riemann's formula to deduce our numerical scheme and to present from the problems mentioned above, we apply the composition rule to $D^{q} x(t)[3,4,5]$, that is

$$
D^{q} x(t)=D\left[D^{-u} x(t)\right]=\frac{x(0)}{\Gamma(u)} t^{u-1}+D^{-u} \dot{x}(t),
$$

where $\dot{x}(t)=D x(t)$ represents the velocity of the oscillator, and $x(0)$ is the value of displacement at $t=0$ and is often given as an initial condition.

The numerical algorithm to calculate the fractional derivative $D^{q} x(t)$ at $t=t_{n}$ of Eq. (27) is

$$
\begin{aligned}
D^{q} x\left(t_{n}\right) & =\frac{x(0)}{\Gamma(1-q)} t_{n}^{-q}+D^{q-1} \dot{x}\left(t_{n}\right) \\
& =\frac{x(0)}{\Gamma(1-q)} t_{n}^{-q}+\frac{1}{\Gamma(1-q)} \int_{0}^{t_{n}} \frac{\dot{x}(\tau)}{\left(t_{n}-\tau\right)^{q}} d \tau \\
& =\frac{1}{\Gamma(1-q)} \frac{x(0)}{t_{n}^{q}}+\frac{1}{\Gamma(1-q)}\left[\int_{0}^{t_{n-1}} \frac{\dot{x}(\tau)}{\left(t_{n}-\tau\right)^{q}} d \tau+\int_{t_{n-1}}^{t_{n}} \frac{\dot{x}(\tau)}{\left(t_{n}-\tau\right)^{q}} d \tau\right],
\end{aligned}
$$

where we denote

$$
I_{0}=\frac{x(0)}{t_{n}^{q}}, \quad I_{n-1}=\int_{0}^{t_{n-1}} \frac{\dot{x}(\tau)}{\left(t_{n}-\tau\right)^{q}} d \tau, \quad \Delta I_{n}=\int_{t_{n-1}}^{t_{n}} \frac{\dot{x}(\tau)}{\left(t_{n}-\tau\right)^{q}} d \tau .
$$

By substituting relationships (29) into (28) we become the following equation

$$
D^{q} x\left(t_{n}\right)=\frac{1}{\Gamma(1-q)}\left(I_{0}+I_{n-1}+\Delta I_{n}\right),
$$

From Eq. (25) we have the following iterative computational scheme

$$
\dddot{x}\left(t_{n}\right)+a \ddot{x}\left(t_{n}\right)+b D^{q} x\left(t_{n}\right)+c x\left(t_{n}\right)=f\left(t_{n}\right),
$$

where $x\left(t_{n}\right)$ and $\ddot{x}\left(t_{n}\right)$ with subscript $n$ denote the displacement and acceleration at time $t_{n}$, respectively.

We approximate the ordinary definite integral $I_{n-1}$ by trapezoid numerical integration as

$$
I_{n-1} \approx \frac{h}{2}\left[\frac{\dot{x}_{0}}{t_{n}^{q}}+\frac{\dot{x}_{n-1}}{h^{q}}+2 \sum_{i=1}^{n-2} \frac{\dot{x}(i h)}{\left(t_{n}-i h\right)^{q}}\right], \quad h=t_{n}-t_{n-1}, n \geq 2 .
$$


Now we note the integrals $\Delta I_{n}$ of equation (29). We must define $\dot{x}(\tau)$ under integral sign to calculate that integral. With $t_{n-1} \leq \tau \leq t_{n}$, we use the Taylor expansion and omit high order terms because the interval $\tau-t_{n-1}$ could be taken very small.

$$
\dot{x}(\tau)=\dot{x}_{n-1}+\left(\tau-t_{n-1}\right) \ddot{x}_{n-1}=\dot{x}_{n-1}+\frac{\left(\tau-t_{n-1}\right)}{h}\left(\dot{x}_{n}-\dot{x}_{n-1}\right),
$$

By substituting (33) into (29), we obtain

$$
\begin{aligned}
\Delta I_{n} & =\int_{t_{n-1}}^{t_{n}} \frac{\dot{x}_{n-1}}{\left(t_{n}-\tau\right)^{q}} d \tau+\int_{t_{n-1}}^{t_{n}} \frac{\dot{x}_{n}-\dot{x}_{n-1}}{h} \cdot \frac{\tau-t_{n-1}}{\left(t_{n}-\tau\right)^{q}} d \tau \\
& =\frac{h^{1-q}}{1-q} \dot{x}_{n-1}+\frac{h^{1-q}}{(1-q)(2-q)}\left(\dot{x}_{n}-\dot{x}_{n-1}\right) .
\end{aligned}
$$

From Eq. (22) we have the Newmark approximation of $\dot{x}_{n}$

$$
\dot{x}_{n}=\frac{\gamma}{\beta h}\left(x_{n}-x_{n-1}\right)+\left(1-\frac{\gamma}{\beta}\right) \dot{x}_{n-1}+\left(1-\frac{\gamma}{2 \beta}\right) h \ddot{x}_{n-1}+\left(\frac{1}{2}-\frac{\gamma}{6 \beta}\right) h^{2} \dddot{x}_{n-1},
$$

From equation (34) and (35), we yield

$$
\begin{aligned}
& \Delta I_{n}=\frac{h^{1-q}}{(1-q)(2-q)} {\left[\frac{\gamma}{\beta h}\left(x_{n}-x_{n-1}\right)+\left(2-q-\frac{\gamma}{\beta}\right) \dot{x}_{n-1}\right.} \\
&\left.+\left(1-\frac{\gamma}{2 \beta}\right) h \ddot{x}_{n-1}+\left(\frac{1}{2}-\frac{\gamma}{6 \beta}\right) h^{2} \dddot{x}_{n-1}\right],
\end{aligned}
$$
as follows

From Eqs. (21) and (23) we have the Newmark approximation formulas for $\dddot{x}_{n}, \ddot{x}_{n}$

$$
\begin{gathered}
\dddot{x}_{n}=\frac{1}{\beta h^{3}}\left(x_{n}-x_{n-1}\right)-\frac{1}{\beta h^{2}} \dot{x}_{n-1}-\frac{1}{2 \beta h} \ddot{x}_{n-1}-\left(\frac{1}{6 \beta}-1\right) \dddot{x}_{n-1}, \\
\ddot{x}_{n}=\frac{\alpha}{\beta h^{2}}\left(x_{n}-x_{n-1}\right)-\frac{\alpha}{\beta h} \dot{x}_{n-1}+\left(1-\frac{\alpha}{2 \beta}\right) \ddot{x}_{n-1}+\left(1-\frac{\alpha}{6 \beta}\right) h \dddot{x}_{n-1} .
\end{gathered}
$$

By substituting Eq. (30) into the differential equation of motion (31) we have

$$
\dddot{x}_{n}+a \ddot{x}_{n}+b \frac{1}{\Gamma(1-q)} \Delta I_{n}+c x_{n}=f\left(t_{n}\right)-b \frac{1}{\Gamma(1-q)}\left(I_{0}+I_{n-1}\right),
$$

From (36), (37) and (38), we can calculate the left side of (39)

$$
\begin{aligned}
& \dddot{x}_{n}+a \ddot{x}_{n}+b \frac{1}{\Gamma(1-q)} \Delta I_{n}+c x_{n}=\left[\frac{1}{\beta h^{3}}+a \frac{\alpha}{\beta h^{2}}+b \frac{h^{-q}}{\Gamma(3-q)} \frac{\gamma}{\beta}+c\right] x_{n} \\
& -\left[\frac{1}{\beta h^{3}} x_{n-1}+\frac{1}{\beta h^{2}} \dot{x}_{n-1}+\frac{1}{2 \beta h} \ddot{x}_{n-1}+\left(\frac{1}{6 \beta}-1\right) \dddot{x}_{n-1}\right]- \\
& a\left[\frac{\alpha}{\beta h^{2}} x_{n-1}+\frac{\alpha}{\beta h} \dot{x}_{n-1}+\left(\frac{\alpha}{2 \beta}-1\right) \ddot{x}_{n-1}+\left(\frac{\alpha}{6 \beta}-1\right) h \dddot{x}_{n-1}\right] \\
& -b \frac{h^{1-q}}{\Gamma(3-q)}\left[\frac{\gamma}{\beta h} x_{n-1}+\left(q-2+\frac{\gamma}{\beta}\right) \dot{x}_{n-1}+\left(\frac{\gamma}{2 \beta}-1\right) h \ddot{x}_{n-1}+\left(\frac{\gamma}{6 \beta}-\frac{1}{2}\right) h^{2} \dddot{x}_{n-1}\right],
\end{aligned}
$$


By substituting Eq.(40) into Eq.(39) we obtain our one-step numerical scheme as

$$
\begin{aligned}
& {\left[\frac{1}{\beta h^{3}}+a \frac{\alpha}{\beta h^{2}}+b \frac{h^{-q}}{\Gamma(3-q)} \frac{\gamma}{\beta}+c\right] x_{n}=f\left(t_{n}\right)-b \frac{1}{\Gamma(1-q)}\left(I_{0}+I_{n-1}\right)} \\
& +\left[\frac{1}{\beta h^{3}} x_{n-1}+\frac{1}{\beta h^{2}} \dot{x}_{n-1}+\frac{1}{2 \beta h} \ddot{x}_{n-1}+\left(\frac{1}{6 \beta}-1\right) \dddot{x}_{n-1}\right] \\
& +a\left[\frac{\alpha}{\beta h^{2}} x_{n-1}+\frac{\alpha}{\beta h} \dot{x}_{n-1}+\left(\frac{\alpha}{2 \beta}-1\right) \ddot{x}_{n-1}+\left(\frac{\alpha}{6 \beta}-1\right) h \dddot{x}_{n-1}\right] \\
& +b \frac{h^{1-q}}{\Gamma(3-q)}\left[\frac{\gamma}{\beta h} x_{n-1}+\left(q-2+\frac{\gamma}{\beta}\right) \dot{x}_{n-1}+\left(\frac{\gamma}{2 \beta}-1\right) h \ddot{x}_{n-1}+\left(\frac{\gamma}{6 \beta}-\frac{1}{2}\right) h^{2} \dddot{x}_{n-1}\right] .
\end{aligned}
$$

We finally obtain numerical solution $x_{n}$ of the differential equation of motion (25) through the values of $x_{n-1}, \dot{x}_{n-1}, \ddot{x}_{n-1}, \dddot{x}_{n-1}$ where $\dot{x}_{n-1}, \ddot{x}_{n-1}$ and $\dddot{x}_{n-1}$ are calculated as follows

$$
\left\{\begin{array}{l}
\dddot{x}_{n}=\frac{1}{\beta h^{3}}\left(x_{n}-x_{n-1}\right)-\frac{1}{\beta h^{2}} \dot{x}_{n-1}-\frac{1}{2 \beta h} \ddot{x}_{n-1}-\left(\frac{1}{6 \beta}-1\right) \dddot{x}_{n-1}, \\
\ddot{x}_{n}=\ddot{x}_{n-1}+(1-\alpha) h \dddot{x}_{n-1}+\alpha h \dddot{x}_{n}, \\
\dot{x}_{n}=\dot{x}_{n-1}+h \ddot{x}_{n-1}+\left(\frac{1}{2}-\gamma\right) h^{2} \dddot{x}_{n-1}+\gamma h^{2} \dddot{x}_{n} .
\end{array}\right.
$$

We suppose that initial conditions to above formulas $x(0), \dot{x}(0)$ and $\ddot{x}(0)$ are given.

\section{NUMERICAL EXAMPLES}

We have tried out the algorithm on some examples. For the first example, we have chosen

$$
\begin{gathered}
f=\sin \left(t+\frac{\pi}{3}\right), \\
a=1.3, \quad b=0.5, \quad c=0.25, \quad q=0.5, \quad h=0.01, \\
\alpha=0.5, \quad \gamma=0.25, \quad \beta=\frac{1}{12}
\end{gathered}
$$

and the initial conditions

$$
\begin{gathered}
x(0)=0, \quad \dot{x}(0)=1, \quad \ddot{x}(0)=0 \\
\Rightarrow \dddot{x}(0)=\frac{\sqrt{3}}{2} .
\end{gathered}
$$

The differential equation of motion has following form

$$
\dddot{x}+1.3 \ddot{x}+0.5 D^{1 / 2} x(t)+0.25 x=\sin \left(t+\frac{\pi}{3}\right) .
$$

The solution of Eq. (43) obtained by the Newmark method is compared in Fig. 1 with the numerical results using the Runge - Kutta - Nystroem method. 


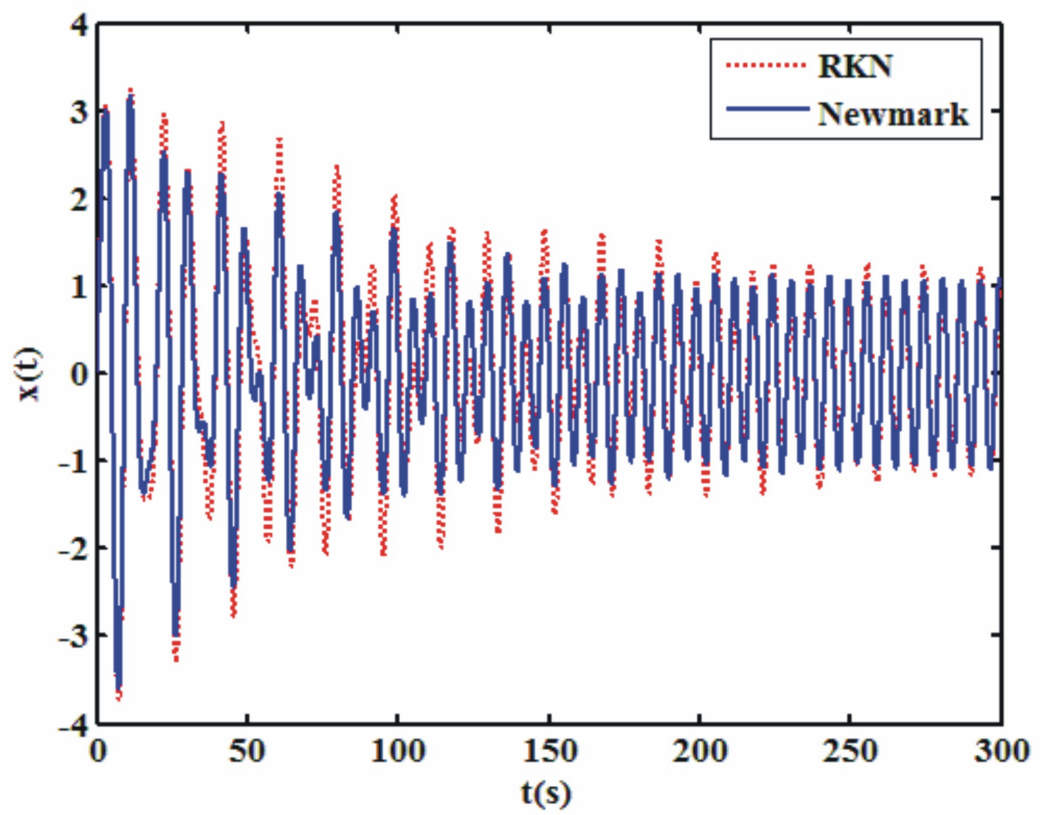

Fig. 1. Time histories of the displacement in Eq. (43) with $h=0.01$.

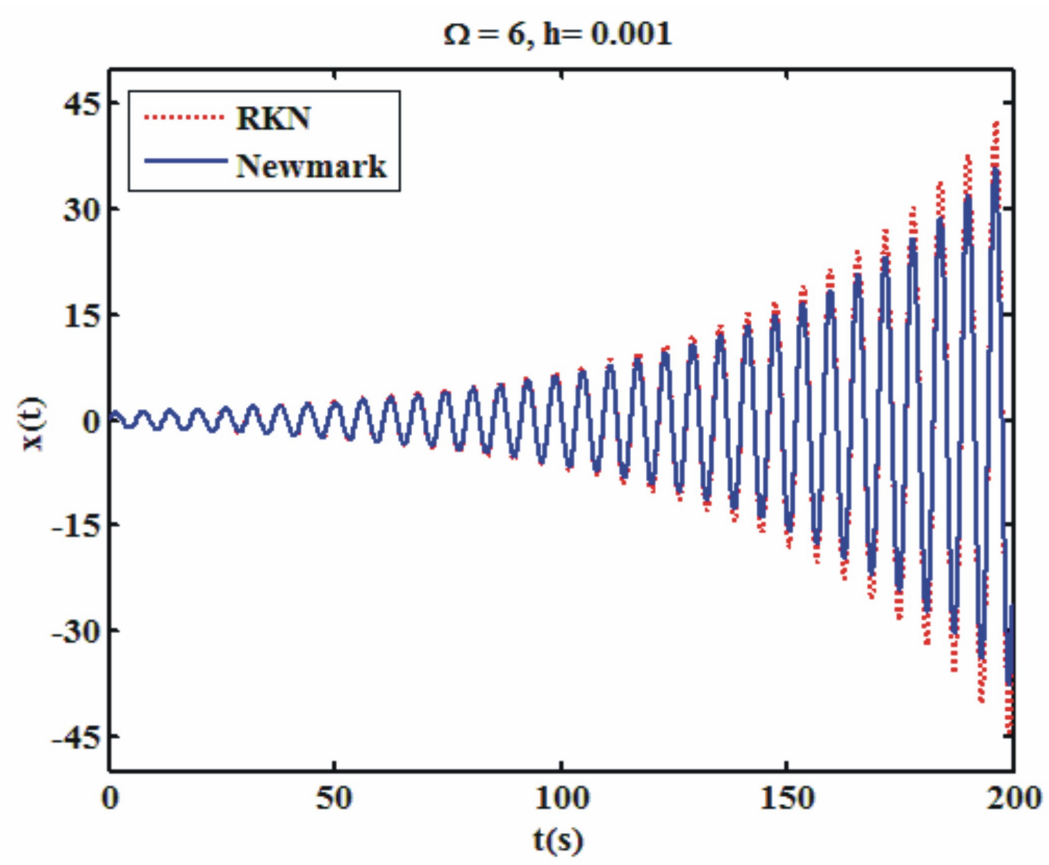

Fig. 2. Time histories of the displacements in Eq. (44) with $h=0.001$. 
In the second example the numerical computations are carried out using the following parameters

$$
f=5 \sin (\Omega t), \quad a=10, \quad b=1, \quad c=10, \quad q=0.5 .
$$

The initial conditions have chosen as

$$
x(0)=0, \quad \dot{x}(0)=1, \quad \ddot{x}(0)=0 \quad \Rightarrow \dddot{x}(0)=0 .
$$

The differential equation of motion of the system in this case has following form

$$
\dddot{x}+10 \ddot{x}+D^{1 / 2} x(t)+10 x=5 \sin (\Omega t) .
$$

The numerical results of Eq. (44) obtained by the Runge-Kutta-Nystroem method and Newmark method are shown in Fig. 2.

\section{CONCLUSIONS}

Based on idea of the Newmark integration method, the approximation formulas for the third order dynamic systems are developed in this paper. Then, using the Liouville - Rieman's definition of fractional derivatives and the Newmark integration scheme, a numerical algorithm is developed to calculate dynamic response of third order systems involving fractional derivatives. In two examples, a good agreement is obtained between the results by the Newmark method and the Runge - Kutta - Nystroem method.

The single - step Newmark numerical integration algorithm presented here for fractional third order systems is effective and successful. According to this algorithm, a computer program is developed using MATLAB software.

\section{ACKNOWLEDGMENT}

This paper was completed with the financial support by the Vietnam National Foundation for Science and Technology Development (NAFOSTED).

\section{REFERENCES}

[1] N. M. Newmark, A Method of Computation for structural Dynamics, ASCE Journal of Engineering Mechanics Division, 85, (1959), 67 - 94.

[2] M. Géradin, D. Rixen, Mechanical Vibrations, Wiley, Chichester, (1994).

[3] K. B. Oldham, J. Spanier, The Fractional Calculus, Academic Press, Boston, New York (1974).

[4] I. Podluny, Fractional Differential Equations, Academic Press, Boston, New York (1999).

[5] K. Miller, B. Ross, An Introduction to the Fractional Calculus and Fractional Differential Equations, John Wiley \& Sons, New York (1993).

[6] W. Zhang, N. Shimizu, Numerical Algorithm for Dynamic Problems Involving Fractional Operator, International Journal of JSME, Series C, 41(3), (1998), 364 - 370.

[7] W. Zhang, N. Shimizu, Damping Properties of the Viscoelastic Material Described by Fractional Kelvin-Voigt Model, International Journal of JSME, Series C, 42(1), (1999), 1-9.

[8] N. Shimizu, W. Zhang, Fractional Calculus Approach to Dynamic Problems of Viscoelastic Materials, International Journal of JSME, Series C, 42(4), (1999), 825 - 837. 